\title{
Clinical and Pathological Aspects of the Sequelae of Urogenital Schistosomiasis: Findings Regarding 43 Cases
}

\author{
Boubacar Fall ${ }^{1}$,, Yaya Sow ${ }^{1}$, Aboubacar Traoré ${ }^{1}$, Cyrille Ze Ondo ${ }^{1}$, Mouhamedou Diagana ${ }^{2}$, \\ Alioune Sarr ${ }^{1}$, Babacar Sine ${ }^{1}$, Samba Thiapato Faye ${ }^{1}$, Modou Ndiaye ${ }^{1}$, Ousmane Sow ${ }^{1}$, \\ Mamadou Ba ${ }^{1}$
}

${ }^{1}$ Department of Urology, Aristide Le-Dantec Hospital, Dakar, Senegal

${ }^{2}$ Department of Urology, Cheikh Zayed Hospital, Nouakchott, Mauritania

Email address:

bbcrfall@yahoo.fr (B. Fall)

${ }^{*}$ Corresponding author

\section{To cite this article:}

Boubacar Fall, Yaya Sow, Aboubacar Traoré, Cyrille Ze Ondo, Mouhamedou Diagana, Alioune Sarr, Babacar Sine, Samba Thiapato Faye, Modou Ndiaye, Ousmane Sow, Mamadou Ba. Clinical and Pathological Aspects of the Sequelae of Urogenital Schistosomiasis: Findings Regarding 43 Cases. International Journal of Clinical Urology. Vol. 3, No. 1, 2019, pp. 10-14. doi: 10.11648/j.ijcu.20190301.13

Received: April 28, 2019; Accepted: June 2, 2019; Published: June 12, 2019

\begin{abstract}
Purpose: To describe the clinical-pathological profile of sequelae of urogenital schistosomiasis. Patients and methods: We performed a retrospective study of the sequelae for cases of urogenital schistosomiasis treated between January 2011 and December 2016. These cases were from Senegal and neighboring countries. Results: We included 43 cases. The mean age of the patients was $43.2 \pm 16.6$ years (14 - 75 years). The sex ratio was 3.3. The sites of the sequelae lesions were the bladder and pelvic ureters in $83.7 \%$, the bladder only in $13.9 \%$, and the ureters only in $2.3 \%$ of the patients. Bladder wall calcification was the most common lesion (74.4\%), followed by bladder masses (48.8\%). Stenosis of the orifice was the most common ureteral lesion (30.2\%). The bladder masses were a squamous cell carcinoma in ten cases, a urothelial carcinoma in one case, and a schistosomiasis granuloma in five cases. In one patient, the histological type was not specified. Of the ten cases of squamous cell carcinoma, eight had died of cancer. Of the twelve cases of ureteral orifice stenosis treated by ureterocystoneostomy, the outcome was good in nine cases and poor in three patients. Conclusion: In this study, sequelae of urogenital schistosomiasis were most often observed in young adult males. The most common sequelae were vesical and ureteral calcifications, bladder cancers, and pelvic ureter strictures.
\end{abstract}

Keywords: Urogenital Schistosomiasis, Calcifications, Bladder Cancer, Urinary Obstruction

\section{Introduction}

Described for the first time by Théodore Bilharz in 1851, Schistosoma hematobium (Sh) is a trematode hematophage responsible for urogenital schistosomiasis (UGS) [1]. It is a parasite of the urinary and genital perivisceral venous plexuses. The laying of eggs by female worms in the submucosa of the targeted organs triggers an inflammatory reaction (granuloma) that develops into fibrosis and/or calcification. These irreversible tissue lesions are observed particularly in case of severe and repeated infections. They can result in stenoses of the excretory tract, progressive alteration of renal function, and bladder cancer [2, 3].

In Senegal, UGB is an endemic and pronounced infection, particularly in children [4]. Despite its endemic nature, there have been few studies on the sequelae lesions and their consequences [5].

The objective of this study was to report the profile of the sequelae from UGB in our area and to evaluate the outcomes of their treatment.

\section{Patients and Methods}

We carried out a retrospective study of the sequelae for cases of urogenital schistosomiasis treated between January 2011 and December 2016. These cases came from Senegal and adjoining countries. The diagnosis of sequelae of urogenital schistosomiasis was often made based on a set of 
very indicative indirect evidence in patients who had a history of spending time in schistosomiasis endemic areas, swimming in freshwater holes, and terminal hematuria. This evidence was based either on medical imaging, cystoscopy, or also by a histological examination (revealing eggs of $\mathrm{SH}$ in biopsies or surgical specimens) $[2,3,6]$. The studied parameters were the following:

1. Epidemiological: age, gender, geographical origin;

2. Clinical: circumstances surrounding the discovery, the type and the location of the sequelar lesions;

3. Therapeutic: the type of treatment carried out, the outcome of the treatment, and changes after the treatment. After ureterocystoneostomy, the outcome (after removal of the ureteral or JJ stent) was considered to be good in the absence of a recurrence of the ureteral obstruction with regression or disappearance of the upstream ureterohydronephrosis.

The data were analyzed with Microsoft Excel 2013 software.

\section{Results}

We included 43 patients. The average age of the patients was $43.2 \pm 16.6$ years $(14-75$ years). The sex ratio $(\mathrm{M} / \mathrm{F})$ was 3.3 .

The sequelar lesions were located both in the bladder and the pelvic ureter in $83.7 \%$ of the cases, only the bladder in $13.9 \%$ of the cases, and only the ureter in $2.3 \%$ of the cases (Table 1). In terms of the bladder, wall calcification (Figure 1) was the most common lesion $(74.4 \%)$, followed by a mass in the bladder (48.8\%) (Figure 2). Stenosis of one or two ureteral orifices was the most common ureteral lesion (Table 2) followed by ureteral stricture (Figure 3 ). A single genital lesion was noted in our patients. It involved calcification of the seminal vesicles in a patient who already had stenosis of the pelvic ureter.

The most commonly carried out treatments were transurethral resection of the bladder in 17 cases $(43.59 \%)$ and ureterocystoneostomy in 12 cases (27.9\%) (Table 3). After transurethral resection of the bladder, histology of the resection shavings revealed a squamous cell carcinoma in 10 cases, a urothelial carcinoma in one case, and a schistosomal granuloma in five cases. For one patient, the histological result had not been reported. No radical cystectomies were carried out due to the advanced stage of the cancers at the time of the diagnosis. Of the ten cases of squamous cell carcinoma, eight had died as a result of their cancer. After ureterocystoneostomy, there was a favorable outcome in nine cases and a poor outcome in three patients with a recurrence of the ureteral obstruction at the level of the intramural ureter and persistence of the ureterohydronephrosis. In the patient who was initially treated by Boari-Küss flap, the stricture of the pelvic ureter recurred and the intervention was resumed with placement of a JJ stent that was changed regularly.

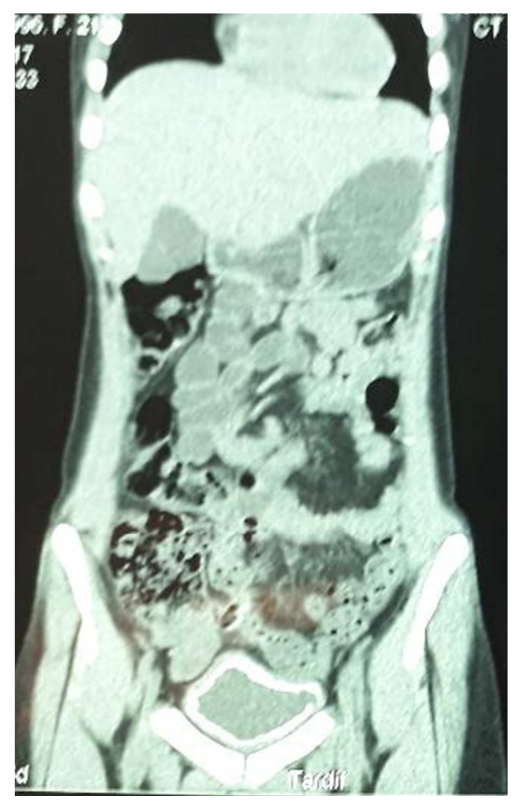

Figure 1. Calcification of the entire bladder wall.

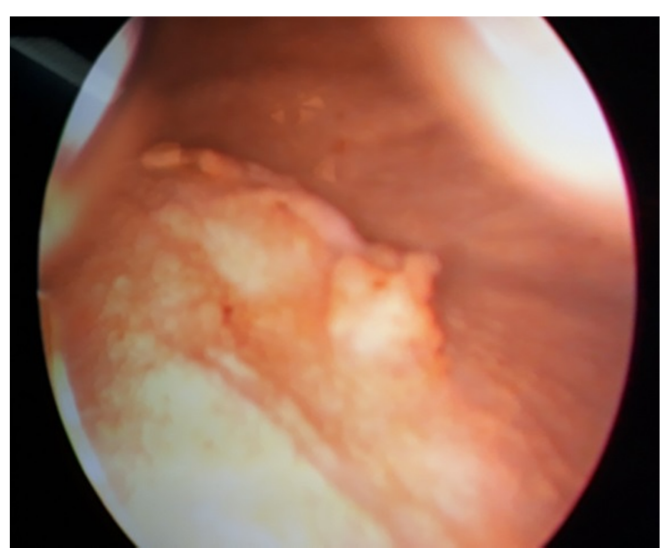

Figure 2. Schistosomal bladder masse around the ureteral orifice.

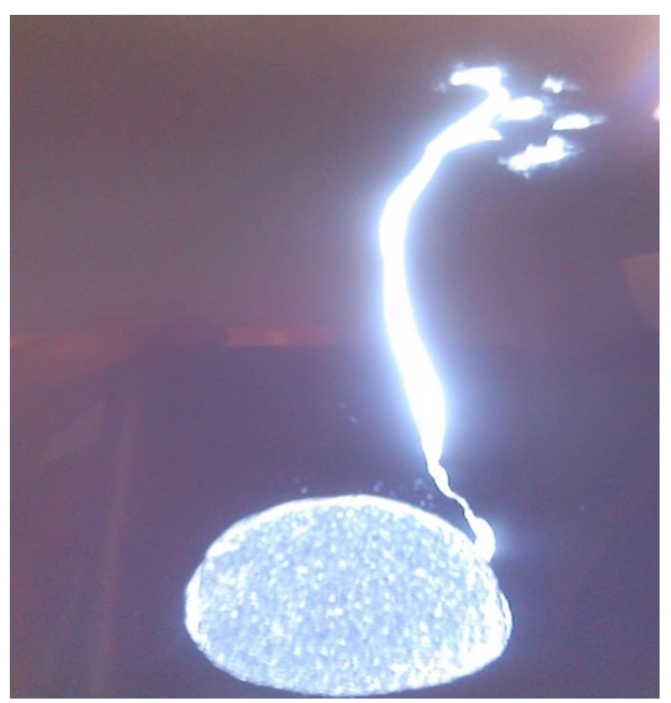

Figure 3. Extensive stricture of the left pelvic ureter on a single anatomical kidney. 
Table 1. Repartition of the patients according to the type of bladder lesion.

\begin{tabular}{lll}
\hline Type of bladder lesion & Number of cases & Percentage (\%) \\
\hline Bladder wall calcifications & 32 & 74.4 \\
Bladder masses & 21 & 48.8 \\
Small-capacity scarred bladder & 13 & 30.2 \\
Sclerosis of the bladder neck & 6 & 13.9 \\
Bladder stones & 3 & 6.9 \\
\hline
\end{tabular}

Table 2. Repartition of the patients according to the type of lesion of the upper urinary system.

\begin{tabular}{lll}
\hline Type of lesion of the upper urinary system & Number of cases & Percentage (\%) \\
\hline Stenosis of ureteral orifices & 13 & 30.2 \\
Single or multiple ureteral strictures & 3 & 6.9 \\
Ureteral stones & 5 & 11.6 \\
Ureteral wall calcifications & 5 & 11.6 \\
Non-functional dilated kidney with ureteral stricture & 1 & 2.3 \\
Obstructive kidney failure & 4 & 9.3 \\
\hline
\end{tabular}

Table 3. Repartition of the patients according to the type of treatment carried out.

\begin{tabular}{lll}
\hline Type of treatment carried out & Number of cases & Percentage (\%) \\
\hline Transurethral bladder resection & 17 & 39.5 \\
Ureterocystoneostomy + J & 6 & 13.9 \\
Ureterocystoneostomy + ureteral stent & 6 & 13.9 \\
Boari- Küss flap + JJ & 1 & 2.3 \\
Segmental resection of the ureter + end-to-end anastomosis with a JJ stent & 1 & 2.3 \\
Placement of a JJ stent & 2 & 4.6 \\
Transileal ureterostomy according to Bricker (for advanced bladder cancer) & 1 & 2.3 \\
Nephrectomy of a non-functional kidney & 1 & 2.3 \\
Open ureterolithotomy & 5 & 11.6 \\
Cystolithotomy + Incision of the bladder neck & 3 & 6.9 \\
\hline
\end{tabular}

\section{Discussion}

Although urogenital schistosomiasis (UGS) can affect the entire urinary tract, it primarily affects specific sites such as the bladder, the pelvic ureter, and the seminal vesicles in men [7]. In our study, the sequelar lesions were simultaneously vesical and ureteral in $83.7 \%$ of the cases. The initial lesion is a reaction of the host against the eggs deposited in the tissues. In the beginning, it manifests as an inflammatory granuloma. Its healing or its progression toward fibrotic and retractile scarring or calcification depends on the number of eggs retained in the tissues [2]. An intermittent infection that is treated early on and in an appropriate manner typically heals without sequelae. A severe infection, frequent reinfections, delayed and/or inadequate treatment, or a lack of treatment leads to the appearance of retractile tissue fibrosis and calcifications due to the eggs retained in the tissues [2].

We encountered twelve cases of bladder cancer, of which ten were squamous cell carcinomas. It has long been known that there is a causal link between UGS and cancer $[8,9]$. The mechanisms leading to the induction of cancer have not yet been properly elucidated. However, the main mechanism is thought to involve chronic inflammation of the bladder wall as a result of the retention of Sh eggs in the tissue [10, 11]. The clinical pathology characteristics of bladder cancers induced by UGS are a young age of the patients, male predominance, a squamous cell carcinoma nature of the carcinomas, frequent intratumoral calcifications, and aggressiveness of the cancers $[10,11]$. These characteristics were noted among our patients. The similarity of the clinical signs of bladder cancer (terminal hematuria and irritative lower urinary system symptoms) with those of progressive urogenital schistosomiasis results in these cancers often being diagnosed at locally advanced or metastatic stages in our context of endemic schistosomiasis. This was the case for all of our patients and the reason why none of them were treated by radical cystectomy. Once such a cystectomy is no longer feasible, the prognosis for the patients is poor as bladder cancer induced by Schistosomiasis are largely refractory to both radiotherapy and chemotherapy [12]. Of the ten cases of squamous cell carcinoma, eight had died as a result of their cancer. In their series, Abdou et al. reported 15 cases of cancer that were mostly at an advanced stage [13]. Seven of these patients underwent a cystectomy and the others received palliative treatment. At five years, seven patients had died and five were lost to follow-up. The high rate of mortality with these cancers, therefore, appears to be due mainly to the delay in diagnosis.

In terms of the bladder, wall calcification was the most frequent lesion (74.4\%). Fibrosis and wall calcification of the bladder alter its dynamics. They can result in hypotonia or an atonic bladder responsible for poor emptying [7]. The ensuing urinary stasis promotes the occurrence of bacterial urinary infections and the induction of bladder cancer such as squamous cell carcinomas in particular [10]. Fibrosis and bladder calcification can also lead to a high-pressure contracted bladder, which constitutes a danger for the upper urinary system. Bladder retraction can justify performing an 
enterocystoplasty, which was not done for our patients [13]. The bladder neck is one of the main sites for the deposition of Sh eggs [7]. The ensuing intense inflammation results in bladder outlet obstruction [3]. Such an obstruction promotes bladder urinary stasis and bacterial urinary infections. We have treated three cases of bladder-neck stenosis by an incision that allows the neck to be enlarged [3]. The risk of recurrence is high, however.

The fibrous and stenosing sequelae are frequently located at the level of the pelvic ureters. They can alter their peristaltic movement and thereby contribute to the creation of a functional obstruction [6]. They can also lead to a stenotic ureteral obstruction. This can result in uni or bilateral ureterohydronephrosis that can progress to alteration of renal function. Strictures of the pelvic ureter are hence potentially serious. They can readily be diagnosed by medical imaging, particularly by tomodensitometric urography $[6,14]$. Praziquantel is effective at the early stage of UGS (arrest of egg-laying, destruction of the parasite, and regression of inflammatory granulomas) but has no effect on the nonreversible stages of fibrosis and calcification [2]. Treatment of obstructive lesions of the pelvic ureter, therefore, calls for open or endoscopic surgery. This can be done by several methods such as high-pressure balloon dilation [15], ureterocystoneostomy or ureteroileoplasty [13]. After balloon dilatation of the inflammatory strictures of the ureter, the rate of long-term recurrence is high and prolonged placement of a JJ stent allows for improvement of the outcomes [15]. We have not used this therapeutic approach with our patients due to the unavailability of the required equipment. However, we did perform a ureterocystoneostomy in twelve cases. It is suitable for treatment of stenosing lesions of the pelvic ureter, and it allows such lesions to be excised and to reestablish ureterovesical continuity. Nonetheless, with UGS, the inflammatory lesions often extend beyond the stenosed ureteral portion. Moreover, the bladder wall (on which the stoma is carried out) is often fibrotic. Anastomosis of the two altered tissues by the same fibrous remnant exposes to recurrence of the stricture [3]. In our series of twelve ureterocystoneostomy, the outcome was favorable in eight cases. Placement of a JJ-stent in six cases for a duration of six weeks after direct ureterocystoneostomy without a submucosal tunnel allowed us to obtain good outcomes [2].

This study shows that sequelae of UGS are frequent and serious. Combating this parasitic disease is based on several approaches, such as raising awareness in the population of its potential seriousness, eradication of the parasite, early diagnosis and treatment particularly in populations at risk, and chemoprevention by mass administration of praziquantel. These actions, which are currently taking place in most of the countries involved need to be pursued and strengthened.

\section{Conclusion}

UGS is one of the neglected tropical diseases that the World Health Organization is seeking to eliminate. In our study, sequelae were most often noted in young adult men. The most common sequelae were vesical and ureteral calcifications, bladder cancer, and pelvic ureter strictures.

\section{Conflict of Interest Statement}

All the authors do not have any possible conflicts of interest.

\section{References}

[1] Ziskind B. Urinary schistosomiasis in ancient Egypt. Nephrol Ther 2009; 5: 658-61. https://doi.org/10.1016/j.nephro.2009.06.001.

[2] Mianné D, Perret JL, Lavilledieu S. Bilharziose urogénitale. Encycl. Méd. Chir. (Elsevier Paris). Néphrologie urologie.18230. A10.1998, 13p.

[3] Karl-Horst Bichler, Ilya Savatovsky and the members of the urinary tract infection (UTI) working group of the guidelines office of the European Association of Urology (EAU): EAU guidelines for the management of urogenital schistosomiasis. Eur Urol 2006; 49: 998-1003. https://doi.org/10.1016/j.eururo.2006.02.022.

[4] Senghor B, Diallo A, Sylla SN, Doucouré S, Ndiath MO, Gaayeb L, et al. Prevalence and intensity of urinary schistosomiasis among school children in the district of Niakhar, region of Fatick, Senegal. Parasit Vectors 2014; 7: 5. https://doi.org/10.1186/1756-3305-7-5.

[5] Diao B, Thiam A, Fall B, Fall PA, Diémé MJ, Ndoye AK, et al. Bladder cancer in Senegal: epidemiological, clinical and histological features. Prog Urol 2008; 18: 445-8. https://doi.org/10.1016/j.purol.2008.04.016.

[6] Shebel HM, Elsayes KM, Abou El Atta HM, Elguindy YM, El-Diasty TA. Genitourinary schistosomiasis: Life cycle and radiologic-pathologic findings. RadioGraphics 2012; 32: 1031-46. https://doi.org/10.1148/rg.324115162.

[7] Barsoum RS. Urinary schistosomiasis. J Adv Res 2013; 4: 453-9. https://doi.org/10.1016/j.jare.2012.08.004.

[8] Ferguson AR. Associated bilharziasis and primary malignant disease of the urinary bladder with observations on a series of forty cases. J Pathol Bacteriol 1911; 16: 76-94.

[9] World Health Organization. Evaluation of carcinogenic risk to humans. Schistosomes, liver flukes and Helicobacter pylori. IARC Monogr 1994; 61: 45 -119.

[10] Berry A, Iriart X, Fillaux J, Magnaval JF. Urinary Schistosomiasis and Cancer. Bull Soc Pathol Exot 2017; 110: $68-75$.

[11] Khaled H. Schistosomiasis and Cancer in Egypt. J Adva Res 2013; 4: 461-6. https://doi.org/10.1016/j.jare.2013.06.007.

[12] Zaghloul MS. Bladder cancer and schistosomiasis. J Egypt Natl Canc Inst 2012; 24: 151-159. https://doi.org/10.1016/j.jnci.2012.08.002.

[13] Abdou A, Tligui M, Le Loup G, Raynal S. A western cohort of urinary schistosomiasis. Prog Urol 2012; 22: 598-601. https://doi.org/10.1016/j.purol.2012.03.004. 
[14] Diallo M, Guindo M, Kane B, et al. Evaluation des séquelles bilharziennes du tractus uro - génital par uro-scanner au CHU Gabriel Traoré Bamako-Mali. J Afr Imag Med 2013; 4: 22935 .
[15] De la Taille A, Ravery V, Hoffmann P, et al. Treatment of ureteral stenosis using high pressure dilatation catheters. Prog Urol 1997; 7: 408-14. 